\title{
Multiple Risk Factors of Candida Albicans Associated Denture Stomatitis
}

\author{
Abbas M Al-Kebsi ${ }^{1}$, Fuad Lutf Al-Motareb ${ }^{2}$, Mohsen Al-Hamzy ${ }^{3}$, Hassan A Al-Shamahy4*, Nesreen F Al- \\ Sanabani ${ }^{1}$ and Hashim Esmail Al-Qassimi ${ }^{1}$ \\ ${ }^{1}$ Department of Prosthodontics, Faculty of Dentistry, Republic of Yemen \\ ${ }^{2}$ Department of Orthodontic Pediatric Dentistry, Faculty of Dentistry, Sana'a, Yemen
}

${ }^{3}$ Department of Conservative Dentistry and Oral Health, Republic of Yemen

${ }^{4}$ Medical Microbiology and Clinical Immunology, Faculty of Medicine and Health Sciences, Republic of Yemen

*Corresponding author: Hassan A Al-Shamahy, Medical Microbiology and Clinical Immunology, Faculty of Medicine and Health Sciences, Republic of Yemen.

Received Date: October 21, 2018

Published Date: November 14, 2018

\begin{abstract}
Background and Objectives: Opportunistic oral fungal infections have spread, especially in denture wearers. Denture stomatitis (DS) is a common inflammatory reaction with multifactorial etiology. It is usually associated with Candida species, particularly Candida albicans, due to its high virulence, ability to adhere and form biofilms on oral cavity tissues and denture surfaces. This study highlights the risk factors of Candida albicans-associated denture stomatitis. The study was carried out at Prosthodontics Departments at the Faculty of Dentistry- Sana'a University, Sana'a city - Yemen.
\end{abstract}

Study design: The study group consisted of 288 denture wearers suffering from DS. Data including demographic data of the patients, clinical information, and risk factors of DS recorded in predesigned questionnaire.

Result: There was a significance association between male $(\mathrm{OR}=2.33, \mathrm{p}<0.001)$, older age patients $(\mathrm{OR}=6.8, \mathrm{p}<0.001)$, denture poor fitness (OR=8.2, $\mathrm{p}<0.001)$, older age denture (OR=14.6, $\mathrm{p}<0.001)$, denture manufacturers under supervision of general dentist $(\mathrm{OR}=2.9, \mathrm{p}<0.001)$, non-ideal denture quality $(\mathrm{OR}=4.8, \mathrm{p}<0.001)$, irregular denture cleaning $(\mathrm{OR}=2.1, \mathrm{p}<0.001)$, and often overnight denture wear (OR=1.7, $\mathrm{p}=0.04)$.

Conclusion: Management of predisposing risk factors is key in order to prevent recurrence of DS. Treatment methods may consist of: treatment of any underlying systemic risk factors, improvement in the fit of existing dentures, replacement of existing dentures, improved denture hygiene.

Keywords: Candida albicans-associated denture stomatitis; Denture; Ds risk factors; Sana'a city; Yemen

\section{Introduction}

Longer life expectancy has led to an increase in the ageing population worldwide. This growth in the number of elderly led to an increase in the number of people requiring removable dentures [1,2]. The current rates of edentulism have been estimated to be between 7 percent and 69 percent of the adult population internationally [3]. Candida is present in the oral cavity in two different forms, as floating planktonic cells (blastopores, blastoconidia) and/or in an organized biofilm. Poor oral hygiene, practices such as failure to remove the denture whilst sleeping and poor denture cleansing allows the accumulation of biofilm [46]. Biofilm is defined as structured microbial community that is attached to a surface, consisting of more than $10^{11}$ cells per gram of dry weight $[4,7]$ and bounded by a self-produced extracellular matrix. Biofilms are created adhering to living tissue such as mucosal surfaces or to abiotic surfaces such as oral prostheses, implanted medical devices, and intravascular catheters [8]. It has been recognized that unlike dental biofilm, the biofilm that forms on denture materials possesses a massive population of yeasts [9]. A spongy denture tissue surface, full of nutritive substances, is an idyllic incubator for species such as Candida albicans [10]. Candida albicans is a normal flora in limited number in the oral cavity of 45$65 \%$ of healthy individuals with a higher prevalence recovered in young adults and children [10]. In denture wearers, the prevalence of Candida raised up to $100 \%$ [7,11-17]. In addition Candida can be opportunistic, which can be explained by the fact that dentures 
reduce the flow of oxygen and saliva to the underlying tissue creating a local acidic and anaerobic micro-environment that favours yeast overgrowth. Additionally, Candida has affinity for the acrylic surface of dentures and non-renewing surfaces such as teeth, dental fillings [17-22]. Surface characteristics of denture base acrylic resins, such as hydrophobicity, have generally been acknowledged to be one of the factors contributing to the adhesion, which is a crucial step in biofilm formation [23-25]. Candida albicans biofilms are frequently associated with the occurrence of denture stomatitis $[20,24,26]$. This study highlights the risk factors of Candida albicans-associated denture stomatitis.

\section{Subjects and Laboratory Methods}

Cross sectional study was carried out in the Prosthodontic department at the Faculty of Dentistry- Sana'a University, from $11 / 2016$ to $2 / 2017$ on 288 Yemeni subjects (males and females), aged more than 35 years old. Data were collected by predesigned questionnaire. Samples were collected by wiping premoistened sterile cotton wool swabs on upper and lower denture fitting surfaces. For mycological investigations each sample was inoculated into both media: Sabouraud's dextrose agar and a differential and selective culture media as Candida Chromogenic media. Plates were incubated aerobically for 48 to 72 hours at $37^{\circ} \mathrm{C}$ and were identified systematically by Colonial morphology, wet mount preparation, Gram's stain and germ tube.

\section{Data Collection}

Data including demographic data of the patients and risk factors of Candida albicans DS. The findings were recorded in a predesigned questionnaire with laboratory results.

\section{Ethical Approval}

We obtained written consent from all cases. Assent was taken from participants before collecting the specimens. The study protocol was reviewed and approved by the Ethics Committee of Sana'a University, Faculty of Medicine and Health Sciences.

\section{Result}

This study was conducted on a total of 288 patients contracting DS attending Prosthetic department at the Faculty of DentistrySana'a University from $11 / 2016$ to $2 / 2017$. Male patients accounted for $59.4 \%$ while female patients accounted for $40.6 \%$ of the total cases. The patient's age ranged from 35-65 years, and most of the patients were at the age group of +65 years, $(32.6 \%)$. There was a significant association between male and high risk of contracting Candida albicans DS (OR=2.33, $\mathrm{CI}=1.4$ to 3.9 , and $p<0.001)$. There was a significant association between older age patients ( $\geq$ mean age $=59.5$ years) and contracting Candida albicans DS (OR=6.8 times, $\mathrm{CI}=3.8-10.8$, with $p<0.001$ ) (Table 1 ).

Table 1: The relationship between sex and age (as risk factors) and Candida albicans stomatitis.

\begin{tabular}{|c|c|c|c|c|c|c|}
\hline & \multicolumn{2}{|c|}{ C. albicans positive $\mathrm{n}=168$} & \multirow{2}{*}{ OR } & \multirow{2}{*}{ CI } & \multirow{2}{*}{$\chi^{2}$} & \multirow{2}{*}{$p$} \\
\hline & No & $\%$ & & & & \\
\hline \multicolumn{7}{|c|}{ Sex } \\
\hline Male $n=171$ & 114 & 66.7 & 2.33 & $1.4-3.9$ & 12.03 & $<0.001$ \\
\hline Female $n=117$ & 54 & 46.2 & 0.43 & $0.2-0.17$ & 12.03 & $<0.001$ \\
\hline \multicolumn{7}{|c|}{ Age } \\
\hline$<$ mean age $n=122$ & 41 & 33.6 & 0.1 & $0.09-0.2$ & 53 & $<0.001$ \\
\hline$\geq$ mean age $n=166$ & 127 & 76.5 & 6.4 & $3.8-10.8$ & 53 & $<0.001$ \\
\hline Total $n=288$ & 168 & 58.3 & & & & \\
\hline
\end{tabular}

Mean age $=59.5$ years

Table 2: The connection between Candidal stomatitis and denture fitness and age of denture.

\begin{tabular}{|c|c|c|c|c|c|c|}
\hline \multirow{2}{*}{ Factors } & \multicolumn{2}{|c|}{ C. albicans positive $n=168$} & \multirow{2}{*}{ OR } & \multirow{2}{*}{ CI } & \multirow{2}{*}{$\chi^{2}$} & \multirow{2}{*}{$p$} \\
\hline & No & $\%$ & & & & \\
\hline \multicolumn{7}{|c|}{ Denture fitness } \\
\hline Good $n=52$ & 22 & 42.3 & 0.4 & $0.2-0.8$ & 6.7 & 0.009 \\
\hline Fair $n=99$ & 46 & 46.5 & 0.5 & $0.3-0.7$ & 8.7 & 0.003 \\
\hline Poor $n=137$ & 100 & 73 & & & & $<0.001$ \\
\hline \multicolumn{7}{|c|}{ Denture age (mean in month=20.2 months) } \\
\hline$<$ mean $n=126$ & 33 & 26.2 & 0.07 & $0.04-0.12$ & 95 & $<0.001$ \\
\hline$\geq$ mean $n=162$ & 135 & 84.4 & 14.6 & $8.2-26$ & 95 & $<0.001$ \\
\hline
\end{tabular}

When denture fitness was considered, there was significance association between the poor fitness and Candida albicans DS (OR=8.2, $\mathrm{CI}=8.2-26$, and $p<0.001$ ) (Table 2). Also, there was a highly significant association between older age denture (mean $\geq 20.2$ months) and contracting Candida albicans stomatitis DS (OR=14.6, CI= 8.2-26, with $p<0.001$ ) (Table 2).
Table 3 shows the association of Candidal stomatitis with multiple risk factors of denture stomatitis. There was risk of contracting DS with denture manufacturers supervision under general dentist $(\mathrm{OR}=2.9, \mathrm{CI}=1.6-5$, with $p<0.001)$, non-ideal denture quality (OR=4.8, $\mathrm{CI}=2.8-8.8$, with $p<0.001)$, irregular denture cleaning (OR=2.1, $\mathrm{CI}=1.3-3.5$, with $p<0.001)$, but no significant 
effect with frequency of denture cleaning per day. Also, there was risk of contracting Candida albicans DS with often overnight denture wear $(\mathrm{OR}=1.7, \mathrm{CI}=1.0-31$, with $p=0.04)$, but no significant association between overnight denture solutions or dental checkup per year and contracting Candida albicans DS.

Table 3: The association of Candidal stomatitis with multiple risk factors of denture stomatitis.

\begin{tabular}{|c|c|c|c|c|c|c|}
\hline \multirow{2}{*}{ Factors } & \multicolumn{2}{|c|}{ C. albicans positive $\mathrm{n}=168$} & \multirow{2}{*}{ OR } & \multirow{2}{*}{ CI } & \multirow{2}{*}{$\chi 2$} & \multirow{2}{*}{$p$} \\
\hline & No & $\%$ & & & & \\
\hline \multicolumn{7}{|c|}{ Denture manufacturers under supervision of } \\
\hline General dentist $n=216$ & 140 & 65 & 2.9 & $1.6-5$ & 14.9 & $<0.001$ \\
\hline Dental consultant $n=72$ & 28 & 38.9 & 0.34 & $0.19-0.5$ & 14.9 & $<0.001$ \\
\hline \multicolumn{7}{|c|}{ Denture quality } \\
\hline Non-ideal $n=212$ & 145 & 68.4 & 4.8 & $2.8-8.8$ & 33.4 & $<0.001$ \\
\hline Ideal n=76 & 23 & 30.3 & 0.2 & $0.11-0.03$ & 33.4 & $<0.001$ \\
\hline \multicolumn{7}{|c|}{ Denture hygiene } \\
\hline Regular cleaning $\mathrm{n}=171$ & 87 & 50.9 & 0.4 & $0.28-0.75$ & 9.4 & 0.001 \\
\hline Irregular cleaning $\mathrm{n}=117$ & 81 & 69.2 & 2.1 & $1.3-3.5$ & 9.4 & 0.001 \\
\hline \multicolumn{7}{|c|}{ Freq of cleaning per day } \\
\hline Once $n=33$ & 18 & 54.5 & 0.8 & $0.3-1.5$ & 0.46 & 0.49 \\
\hline Twice $n=43$ & 25 & 55.5 & 0.9 & $0.5-1.9$ & 0.008 & 0.97 \\
\hline Three $n=67$ & 31 & 46.3 & 0.52 & $0.3-0.9$ & 5.2 & 0.02 \\
\hline More $n=28$ & 13 & 46.4 & 0.6 & $0.2-1.2$ & 1.8 & 0.17 \\
\hline \multicolumn{7}{|c|}{ Often Overnight denture wear } \\
\hline Yes $n=75$ & 51 & 68 & 1.7 & $1.0-31$ & 3.9 & 0.04 \\
\hline No $n=213$ & 117 & 54.9 & 0.6 & $0.3-0.9$ & 3.9 & 0.04 \\
\hline \multicolumn{7}{|c|}{ Overnight denture solution } \\
\hline Water $n=151$ & 80 & 53 & 0.6 & $0.3-1.0$ & 3.8 & 0.05 \\
\hline Saline $n=13$ & 6 & 46.1 & 0.6 & $0.19-1.8$ & 0.8 & 0.36 \\
\hline None $\mathrm{n}=49$ & 31 & 63.2 & 1.2 & $0.6-2.4$ & 0.59 & 0.44 \\
\hline \multicolumn{7}{|c|}{ Dental checkup per year } \\
\hline Yes $n=25$ & 11 & 44 & 0.5 & $0.2-1.2$ & 2.3 & 0.12 \\
\hline No $n=263$ & 157 & 59.7 & 1.9 & $0.8-4.3$ & 2.3 & 0.12 \\
\hline
\end{tabular}

\section{Discussion}

In the current study the rate of Candida albicans DS was 58.3\%. This high rate reflects the power of Candida albicans to form biofilm on the denture. There are many factors affect adhesion and biofilm formation of Candida on denture surfaces, such as surface roughness of the inner surface of the prosthesis, salivary pellicle, hydrophobic and electrostatic interactions, receptor-ligand binding $[1,7,15,22,23,28,29]$. Also, there are several reports to suggest the relationship between surface roughness and Candida albicans adherence to denture materials. Huh et al. and Hahnel et al. reported that significantly higher number of Candida albicans was observed on roughened than on smooth surfaces $[22,28]$. Denture base cracks may become to be one of the best sites for microorganism propagation and provides protection from shear forces, even during denture cleaning $[29,30]$. The second step in DS is adherence of Candida albicans to host epithelial cells is a critical first step in the infection process [6-8,23,29-33]. It is essential for both colonization and subsequent induction of mucosal disease [31]. These microorganisms can stick and proliferate through the hard and soft tissues of the oral cavity [29]. Also, the fact that both Candida and epithelial cell surface are negatively charged means that there are disgusting forces retarding their adhesion. Nevertheless, there are other attractive forces such as Lifshitz-van der Waals forces, hydrophobic interactions, and Brownian movement forces. The sum of these nonspecific forces will determine whether the initial nonspecific adhesion between fungal and epithelial cells will be established $[8,23]$.

When gender of patients in the current study was considered, there was significance association between the male patients and risk of increase Candida albicans DS ( $\mathrm{OR}=2.33, \mathrm{CI}=1.4-3.9$, and $p<0.001$ ) (Table 1). Our result is in contrast with previous reports by Chopde et al., Javed et al. and Naik \& Pai in which they have been reported that oral Candida colonization is significantly higher in females compared to males; however, this relationship remains controversial $[16,34,35]$. The Candida colonization lower rate in females compared to males in our study might be explained by the findings of Li-Hui et al. in which salivary $\mathrm{pH}$ values were significantly lower in females than that in males (acidic salivary $\mathrm{pH}$ is unfavorable condition growth for Candida albicans) [36]. When age was taken into account in the current study, there was a highly significant association between older age group ( $\geq$ mean age $=59.5$ years) and contracting Candida albicans DS (OR=6.8 times, $\mathrm{CI}=3.8$ - 
10.8, with $p<0.001$ ) (Table 1). This result can be explained by the fact that older age people generally suffer from systemic illnesses, changes in nourishment and their salivary characteristics as low saliva production and contents [16]. Advancing age is also a risk factor for denture stomatitis in the elderly, because cell-mediated immunity, which provides protection against Candida albicans infection declines with age. According to Ryu et al., some oral environmental factors, such as un-stimulated salivary flow rate and age of subjects, are associated with higher numbers of microbes in the saliva of complete denture wearers. Those authors suggest that a reduction in the salivary flow rate with aging induces an increase in concentration of microbes in saliva [37].

As denture fitness was considered, there was significance association between the poor fitness and Candida albicans DS $(\mathrm{OR}=8.2, \mathrm{CI}=8.2-26$, and $p<0.001)$ (Table 2). An ill-fitting denture may cause frictional irritation of the palatal mucosa and this facilitates invasion of Candida into the superficial layers of the epithelium. On occasions, a denture soft liner may be used to cushion the hard-acrylic material of the denture against the mucosa. Unfortunately, silicone rubber (the most frequently employed material for soft liners) is also a surface that Candida can readily colonize and actually invade [5]. The fact that the oral epithelium is continually replenished means that, in order to colonize the oral mucosa, Candida must be present in the mouth in sufficient numbers and with a high enough growth rate to allow their continued persistence [5].

Once age of the denture was considered in the present study, there was a highly significant association between older age denture ( $\geq$ mean denture age $=20.2$ months) and contracting Candida albicans DS (OR=14.6 times, $\mathrm{CI}=8.2-26$, with $p<0.001)$ (Table 2). This result can be explained by that, denture age is shown to be an important factor as a result of poor fit, roughness, inadequate hygiene, and accumulation of plaque due to aging of denture $[20,25,35]$. It was reported, that aging of the denture and release of residual monomer with time results in poorer fit which affects the contamination of the denture. Moreover, denture age was proportional to Candida colonization and not to degree of inflammation $[11,35]$. Only $25 \%$ of individuals using dentures for $<1$ year were diagnosed with denture stomatitis, whereas $>84 \%$ of those using dentures for $>5$ years had the disease [33].

As soon as oral/denture hygiene was determine, there was significance association between the negative response of cleaning and Candida albicans DS (OR=2.1, CI=1.-3.5, and $p=0.001$ ) (Table $3)$. The present study result is similar to that reported previously in which the negative response of cleaning lead to accumulation of microbial plaque on the surface of the denture in contact with the mucosa [7]. Both the plaque accumulated on the denture and the poor oral hygiene contribute to the virulence of Candida, offering the clinical picture of Candida-associated denture stomatitis $[7,12,16,17,20,25,35-40]$.

In the current study denture wear at night was taken into account as one of predisposing factor of DS (OR=1.7, $\mathrm{p}=0.04)$ (Table 3). Our result is similar to that reported by Chopde et al. and Brondani et al. in which denture wear at night was considered as one of predisposing factor of DS [16,39]. When denture manufacturers supervision was measured, there was significance association between denture prepared under supervision of general dentist and Candida albicans DS (OR=2.9, CI=1.6-5, and $p<0.001$ ) while this risk is absent with denture prepared under supervision of dental consultant (Table 3). Furthermore, there was significance association between non-ideal denture and Candida albicans DS $(\mathrm{OR}=4.8, p<0.001)$. Whether denture manufacturers and denture quality should be considered as predisposing factors are still a matter of debate. Several previous studies have reported that bad denture manufacture and non-ideal denture quality, either alone or in combination with other systemic or local factors, is associated with changes in the oral cavity, increased oral Candida colonization or with the development of oral candidosis.

\section{Conclusion}

Candida albicans DS is a condition that usually affects denture wearers and should be treated in anticipation of asymptomatic. DS needs a shared treatment method from both patient and clinician, and the role of the patient must be stressed. Management of predisposing risk factors is key in order to prevent recurrence. Treatment methods may consist of: treatment of any underlying systemic risk factors, improvement in the fit of existing dentures, replacement of existing dentures, improved denture hygiene. Either methods are in work, the main aim of treatment is to eradicate the biofilm from the patient's dentures. Habitual examination of patients suffering from DS is essential in order to guarantee longterm successful treatment of the situation.

\section{Acknowledgement}

Authors acknowledge the financial support of Sana'a University, Yemen.

\section{Conflict of Interest}

No conflict of interest associated with this work.

\section{References}

1. Coenye T, Prijck KD, Nailis H, Nelis HJ (2011) Prevention of Candida albicans biofilm formation. Open Mycol J 5: 9-20.

2. Yasui M, Ryu M, Sakurai K, Ishihara K (2012) Colonization of the oral cavity by periodontopathic bacteria in complete denture wearers. Gerodontology 29(2): e494-e502.

3. Felton D, Cooper L, Duqum I, Minsley G, Guckes A, et al. (2011) Evidencebased guidelines for the care and maintenance of complete dentures: A publication of the American college of prosthodontists. J Am Dent Assoc 20 Suppl 1: S1-S12.

4. Cruz PC, Andrare IM, Peracini A, Souza-Gugelmin MC, Silva-Lovato $\mathrm{CH}$, et al. (2011) The effectiveness of chemical denture cleansers and ultrasonic device in biofilm removal from complete dentures. J Appl Oral Sci 19(6): 668-673

5. Williams DW, Kuriyama T, Silva S, Malic S, Lewis MA (2011) Candida biofilms and oral candidosis: treatment and prevention. Periodontology 2000 55(1): 250-265.

6. Bhat V, Sharma SM, Shetty V, Shastry CS, Rao V, et al. (2013) Prevalence of Candida associated denture stomatitis (CADS) and speciation of Candida among complete denture wearers of south west coastal region of Karnataka. NUJHS 3(3): 59-63.

7. Salerno C, Pascale M, Contaldo M, Esposito V, Busciolano M, et al. (2011) Candida-associated denture stomatitis. Med Oral Patol Oral Cir Bucal 16(2): 139-143.

8. Darwazeh AMG, Darwazeh TA (2014) What makes oral Candidiasis recurrent infection? a clinical view. J Mycol 2014: 1-5. 
9. Offenbacher S, Barros SP, Altarawneh S, Beck JD, Loewy ZG (2012) Impact of tooth loss on oral and systemic health. Gen Dent 60(6): 494500 .

10. Acosta-Torres LS, Lopez-Marin LM, Nunez-Anita RE, Hernandez-Padron G, Castano VM (2011) Biocompatible metal-oxide nanoparticles: nanotechnology improvement of conventional prosthetic acrylic resins. J Nanomaterials, Vol 2011.

11. Zomorodian K, Haghighi NN, Rajaee N, Pakshir K, Tarazooie B, et al. (2011) Assessment of Candida species colonization and denture-related stomatitis in complete denture wearers. Med Mycol 49(2): 208-211.

12. Ghani F, Chughtai MA, Shah SA (2011) Biochemically assessed pathological activity of oral Candida in denture and non denture wearers. J Postgrad Med Inst 25: 188-198.

13. Abaci O, Haliki-Uztan A (2011) Investigation of the susceptibility of Candida species isolated from denture wearers to different antifungal antibiotics. Afr J Microbiol Res 5: 1398-1403.

14. Kanaguchi N, Narisawa N, Ito T, Kinoshita Y, Kusumoto Y, et al. (2012) Effects of salivary protein flow and indigenous microorganisms on initial colonization of Candida albicans in an in vivo model. BMC Oral Health 12: 36 .

15. Loster BW, Loster J, Wieczorek A, Ryniewicz W (2012) Mycological analysis of the oral cavity of patients using acrylic removable dentures. Gastroenterol Res Pract 2012: 951572.

16. Chopde N, Jawale B, Pharande A, Chaudhari L, Hiremath V, et al. (2012) Microbial colonization and their relation with potential cofactors in patients with denture stomatitis. J Contemp Dent Pract 13(4): 456-459.

17. Sampaio-Maia B, Figueiral MH, Sousa-Rodrigues P, Fernandes MH, Scully C (2012) The effect of denture adhesives on Candida albicans growth in vitro. Gerodontology 29(2): e348-e356.

18. Salim N, Moore C, Silikas N, Satterthwaite J, Rautemaa R (2013) Candidacidal effect of fluconazole and chlorhexidine released from acrylic polymer. J Antimicrob Chemother 68(3): 587-592.

19. Marra J, Paleari AG, Rodriguez LS, Leite ARP, Pero AC, et al. (2012) Effect of an acrylic resin combined with an antimicrobial polymer on biofilm formation. J Appl Oral Sci 20(6): 643-648.

20. Dantas APFM, Consani RLX, Sardi JCO, Mesquita MF (2014) Biofilm formation in denture base acrylic resins and disinfection method using microwave. J Res Pract Dent 2014: 112424.

21. Nam KY, Lee CH, Lee CJ (2012) Antifungal and physical characteristics of modified denture base acrylic incorporated with silver nanoparticles. Gerodontology 29(2): e413-e419.

22. Hahnel S, Rosentritt M, Burgers R, Handel G, Lang R (2012) Candida albicans biofilm formation on soft denture liners and efficacy of cleaning protocols. Gerodontology 29(2): e383-e391.

23. Hoshing C, Dixit S, Mootha A, Diwan N (2011) Role of Candida albicans in denture stomatitis. J Indian Acad Oral Med Radiol 23: 617-619.

24. Lazarin AA, Zamperini CA, Vergani CE, Wady AF, Giampaolo ET, et al. (2014) Candida albicans adherence to an acrylic resin modified by experimental photopolymerised coatings: an in vitro study. Gerodontology 31(1): 25-33.
25. Mima EG, Vergani CE, Machado AL, Massucato EM, Colombo AL, et al. (2012) Comparison of photodynamic therapy versus conventional antifungal therapy for the treatment of denture stomatitis: a randomized clinical trial. Clin Microbiol Infect 18(10): 380-388.

26. Darwazeh AM, Al-Dwairi ZN, Al-Zwairi AA (2010) The Relationship between tobacco smoking and oral colonization with Candida species. J Contemp Dent Pract 11(3): 017-024.

27. Dandekeri S, Prasad K, Shetty M, Hegde C, Sowmya MK, et al. (2013) Occurrence of streptococcus and Candida species and salivary ph in patients wearing complete denture. Int J Health Rehabil Sci 2(4): 198203.

28. Huh JB, Lim Y, Youn HI, Chang BM, Lee JY, et al. (2014) Effect of denture cleansers on Candida albicans biofilm formation over resilient liners. J Adv Prosthodont 6(2): 109-114.

29. Petrovic M, Kostic M, Kostic M, Krunic N, Igic M, et al. (2014) Therapeutic alternatives of natural compounds in treatment of Candida-associated denture stomatitis. Acta Med Medianae 53(1): 73-79.

30. Sahin C, Ergin A, Ayyildiz S, Cosgun E, Uzun G (2013) Effect of biofilm formation, and biocorrosion on denture base fractures. J Adv Prosthodont 5(2): 140-146.

31. Zhu1 W, Filler SG (2010) Interactions of Candida albicans with epithelial cells. Cell Microbiol 12(3): 273-282.

32. Abraham CM (2011) Advances and emerging techniques in the identification, diagnosis and treatment of oral candidiasis. Open Path J 5(9): 8-12.

33. Silva MM, Mima EGO, Colombo AL, Sanita PV, Jorge JH, et al. (2012) Comparison of denture microwave disinfection and conventional antifungal therapy in the treatment of denture stomatitis: a randomized clinical study. Oral Surg Oral Med Oral Pathol Oral Radiol 114(4): 469479 .

34. Javed F, Klingspor L, Sundin U, Altamash M, Klinge B, et al. (2009) Periodontal conditions, oral Candida albicans and salivary proteins in type 2 diabetic subjects with emphasis on gender. BMC Oral Health 9: 12.

35. Naik AV, Pai RC (2011) A Study of factors contributing to denture stomatitis in a North Indian Community. Int J Dent 2011: 589064.

36. Li-Hui W, Chuan-Quan L, Long Y, Ru-Liu L, Long-Hui C, et al. (2016) Gender differences in the saliva of young healthy subjects before and after citric acid stimulation. Clin Chim Acta 460: 142-145.

37. Mima EG, Pavarina AC, Silva MM, Ribeiro DG, Vergani CE, et al. (2011) Denture stomatitis treated with photodynamic therapy: five cases. Oral Surg Oral Med Oral Pathol Oral Radiol Endod 112(5): 602-608.

38. Hoshi N, Mori H, Taguchi H, Taniguchi M, Aoki H, et al. (2011) Management of oral candidiasis in denture wearers. J Prosthodont Res 55(1): 48-52.

39. Brondani MA, Samim F, Feng H (2012) A conventional microwave oven for denture cleaning: a critical review. Gerodontology 29(2): e6-e15.

40. Bakhshi M, Taheri JB, Shabestari SB, Tanik A, Pahlevan R (2012) Comparison of therapeutic effect of aqueous extract of garlic and nystatin mouthwash in denture stomatitis. Gerodontology 29(2): e680-e684. 